\title{
Correction to: Molecular identification and antifungal susceptibility profile of yeast from vulvovaginal candidiasis
}

Yu Shi ${ }^{1,2,3+}$, Yuxia Zhu ${ }^{1,2+}$, Shangrong Fan ${ }^{1,2,3^{*}}$, Xiaoping Liu ${ }^{4}$, Yiheng Liang ${ }^{1,2}$ and Yingying Shan ${ }^{1,2}$

\section{Correction to: BMC Infectious Diseases (2020) 20:287} https://doi.org/10.1186/s12879-020-04985-w

Following publication of the original article [1], the authors identified an error in Table 3. The correct table (Table 3) is given in this erratum and the original article has been corrected.

The original article can be found online at https://doi.org/10.1186/s12879020-04985-w.

*Correspondence: fanshangrong@163.com

${ }^{\dagger}$ Yu Shi and Yuxia Zhu contributed equally to this work

1 Department of Obstetrics and Gynecology, Peking University Shenzhen

Hospital, Shenzhen 518036, China

Full list of author information is available at the end of the article

(c) The Author(s) 2021. Open Access This article is licensed under a Creative Commons Attribution 4.0 International License, which permits use, sharing, adaptation, distribution and reproduction in any medium or format, as long as you give appropriate credit to the original author(s) and the source, provide a link to the Creative Commons licence, and indicate if changes were made. The images or other third party material in this article are included in the article's Creative Commons licence, unless indicated otherwise in a credit line to the material. If material is not included in the article's Creative Commons licence and your intended use is not permitted by statutory regulation or exceeds the permitted use, you will need to obtain permission directly from the copyright holder. To view a copy of this licence, visit http://creativecommons.org/licenses/by/4.0/. The Creative Commons Public Domain Dedication waiver (http://creativeco mmons.org/publicdomain/zero/1.0/) applies to the data made available in this article, unless otherwise stated in a credit line to the data. 
Table 3 In vitro antifungal susceptibility of 1844 clinical isolates of Candida species as determined by the CLSI method

\begin{tabular}{|c|c|c|c|c|c|c|c|c|}
\hline \multirow[t]{2}{*}{ Candida species (n) } & \multicolumn{8}{|c|}{ Antifungal agents } \\
\hline & & BUC & CLO & FLC & ITC & MIC & TEC & VRC \\
\hline C. lusitaniae, $n=1$ & Range & 0.03 & 0.03 & 0.125 & 0.03 & 0.03 & 0.03 & 0.03 \\
\hline \multirow[t]{3}{*}{ C. Fabianii, $n=3$} & Range & $0.03-0.25$ & $0.03-0.06$ & $0.5-1$ & $0.03-0.25$ & $0.125-1$ & 0.03 & 0.03 \\
\hline & MIC50 & 0.125 & 0.03 & 0.5 & 0.06 & 0.125 & 0.03 & 0.03 \\
\hline & MIC90 & 0.25 & 0.06 & 1 & 0.25 & 1 & 0.03 & 0.03 \\
\hline Trichosporon asahii, $n=1$ & Range & 0.03 & 0.03 & 0.25 & 0.03 & 0.06 & 0.03 & 0.03 \\
\hline \multirow[t]{3}{*}{ Rhodotorula, $n=3$} & Range & $0.03-0.5$ & $0.03-1$ & $4-128$ & $0.03-8$ & $0.25-8$ & $0.03-0.5$ & $0.03-1$ \\
\hline & MIC50 & 0.06 & 0.06 & 64 & 2 & 1 & 0.06 & 0.03 \\
\hline & MIC90 & 0.5 & 1 & 128 & 8 & 8 & 0.5 & 1 \\
\hline \multirow[t]{3}{*}{ Kodamaea ohmeri, $n=2$} & Range & $0.125-0.5$ & 0.03 & $0.25-2$ & $0.125-0.25$ & $0.25-0.5$ & 0.03 & 0.03 \\
\hline & MIC50 & 0.125 & 0.03 & 0.25 & 0.125 & 0.25 & 0.03 & 0.03 \\
\hline & MIC90 & 0.5 & 0.03 & 2 & 0.25 & 0.5 & 0.03 & 0.03 \\
\hline \multirow{3}{*}{ Issatchenkia terricola, $n=2$} & Range & $1-4$ & $0.06-0.125$ & $32-64$ & $0.25-0.5$ & 0.5 & 0.25 & 0.25 \\
\hline & MIC50 & 1 & 0.06 & 32 & 0.25 & 0.5 & 0.25 & 0.25 \\
\hline & MIC90 & 4 & 0.125 & 64 & 0.5 & 0.5 & 0.25 & 0.25 \\
\hline Torulaspora pretoriensis, $n=1$ & Range & 0.25 & 0.03 & 8 & 0.5 & 0.5 & 0.125 & 0.125 \\
\hline \multirow[t]{3}{*}{ ATCC90028* } & Range & $0.015-0.5$ & $0.015-0.5$ & $0.125-2$ & $0.015-4$ & $0.008-0.015$ & $0.015-32$ & $0.015-8$ \\
\hline & GM & 0.04 & 0.03 & 0.21 & 0.08 & 0.06 & 0.03 & 0.03 \\
\hline & MIC90 & 0.125 & 0.03 & 0.5 & 0.25 & 0.015 & 0.03 & 0.03 \\
\hline \multirow[t]{2}{*}{ Candida species (n) } & \multicolumn{8}{|c|}{ Antifungal agents } \\
\hline & & $A m B$ & FLU & NYS & TEB & AFG & CFG & MFG \\
\hline \multirow{4}{*}{$\begin{array}{l}\text { C. albicans } \\
n=1272\end{array}$} & Range & $0.015-32$ & $0.03-128$ & $0.03-32$ & $0.03-256$ & $0.008-0.5$ & $0.008-0.5$ & $0.008-0.5$ \\
\hline & GM & 0.22 & 0.70 & 1.60 & 45.11 & 0.015 & 0.1 & 0.03 \\
\hline & MIC90 & 0.5 & 4 & 8 & 256 & 0.03 & 0.25 & 0.25 \\
\hline & $\mathrm{R}$ & & $3.3 \%$ & & & 0 & 0 & 0 \\
\hline \multirow{3}{*}{$\begin{array}{l}\text { C. africana } \\
n=49\end{array}$} & Range & $0.03-32$ & $0.06-8$ & $0.125-4$ & $0.25-256$ & $0.008-0.03$ & $0.015-0.5$ & $0.008-0.5$ \\
\hline & GM & 0.08 & 0.68 & 0.5 & 17.31 & 0.01 & 0.06 & 0.02 \\
\hline & MIC90 & 1 & 2 & 4 & 128 & 0.015 & 0.25 & 0.06 \\
\hline $\begin{array}{l}\text { C. dubliniensis } \\
n=1\end{array}$ & Range & 0.06 & 0.06 & 0.25 & 16 & 0.008 & 0.015 & 0.008 \\
\hline \multirow{4}{*}{$\begin{array}{l}\text { C. glabrata } \\
n=267\end{array}$} & Range & $0.03-2$ & $0.06-16$ & $0.03-32$ & $0.25-256$ & $0.008-0.5$ & $0.008-0.5$ & $0.008-0.5$ \\
\hline & GM & 0.29 & 0.18 & 3.39 & 26.62 & 0.03 & 0.11 & 0.05 \\
\hline & MIC90 & 1 & 1 & 8 & 256 & 0.06 & 0.25 & 0.25 \\
\hline & $\mathrm{R}$ & & 0 & & & 0 & 0 & 0 \\
\hline \multirow{3}{*}{$\begin{array}{l}\text { C. nivariensis } \\
n=9\end{array}$} & Range & $0.06-2$ & $0.125-4$ & $0.5-4$ & $1-256$ & $0.015-0.06$ & $0.08-0.5$ & $0.015-0.5$ \\
\hline & MIC50 & 0.06 & 0.5 & 1 & 128 & 0.06 & 0.25 & 0.015 \\
\hline & MIC90 & 2 & 2 & 4 & 256 & 0.06 & 0.5 & 0.5 \\
\hline \multirow{3}{*}{$\begin{array}{l}\text { C. bracarensis } \\
n=2\end{array}$} & Range & $0.06-1$ & $0.125-2$ & $0.25-8$ & $8-256$ & $0.015-0.03$ & $0.125-0.5$ & $0.015-0.5$ \\
\hline & MIC50 & 0.06 & 0.125 & 0.25 & 8 & 0.015 & 0.125 & 0.015 \\
\hline & MIC90 & 1 & 2 & 8 & 256 & 0.03 & 0.5 & 0.5 \\
\hline \multirow{4}{*}{$\begin{array}{l}\text { C. parapsilosis } \\
\mathrm{n}=76\end{array}$} & Range & $0.03-2$ & $0.125-8$ & $0.03-32$ & $0.25-256$ & $0.008-1$ & $0.008-1$ & $0.008-1$ \\
\hline & GM & 0.19 & 0.14 & 0.59 & 0.62 & 0.69 & 0.60 & 0.54 \\
\hline & MIC90 & 1 & 0.125 & 4 & 32 & 0.5 & 0.5 & 0.5 \\
\hline & $\mathrm{R}$ & & 0 & & & $5.2 \%$ & $5.2 \%$ & $1.3 \%$ \\
\hline
\end{tabular}


Table 3 (continued)

\begin{tabular}{|c|c|c|c|c|c|c|c|c|}
\hline \multirow[t]{2}{*}{ Candida species (n) } & \multicolumn{8}{|c|}{ Antifungal agents } \\
\hline & & $A m B$ & FLU & NYS & TEB & AFG & CFG & MFG \\
\hline \multirow{3}{*}{$\begin{array}{l}\text { C. metapsilosis } \\
\mathrm{n}=20\end{array}$} & Range & $0.015-0.5$ & $0.125-4$ & $0.06-4$ & $0.25-256$ & $0.015-0.5$ & $0.008-0.5$ & $0.015-1$ \\
\hline & GM & 0.10 & 0.177 & 0.46 & 2.17 & 0.17 & 0.17 & 0.39 \\
\hline & MIC90 & 0.5 & 1 & 4 & 256 & 0.25 & 0.25 & 0.5 \\
\hline \multirow[t]{2}{*}{ Candida species (n) } & \multicolumn{8}{|c|}{ Antifungal agents } \\
\hline & & $A m B$ & FLU & NYS & TEB & AFG & CFG & MFG \\
\hline \multirow{3}{*}{$\begin{array}{l}\text { C. orthopsilosis } \\
n=6\end{array}$} & Range & $0.06-0.25$ & $0.125-2$ & $0.06-8$ & $0.25-128$ & $0.008-1$ & $0.015-1$ & $0.008-0.5$ \\
\hline & MIC50 & 0.125 & 1 & 0.5 & 64 & 0.08 & 0.30 & 0.25 \\
\hline & MIC90 & 0.25 & 2 & 8 & 128 & 1 & 1 & 0.5 \\
\hline \multirow{4}{*}{$\begin{array}{l}\text { C. tropicalis } \\
n=61\end{array}$} & Range & $0.03-1$ & $0.125-32$ & $0.03-8$ & $0.25-256$ & $0.015-0.125$ & $0.008-0.5$ & $0.008-0.5$ \\
\hline & GM & 0.19 & 0.23 & 0.54 & 60.02 & 0.03 & 0.24 & 0.04 \\
\hline & MIC90 & 0.5 & 1 & 4 & 256 & 0.06 & 0.5 & 0.5 \\
\hline & $\mathrm{R}$ & & $1.8 \%$ & & & 0 & 0 & 0 \\
\hline \multirow{4}{*}{$\begin{array}{l}\text { C. krusei } \\
n=54\end{array}$} & Range & $0.03-1$ & $0.125-32$ & $0.03-4$ & $16-256$ & $0.015-0.5$ & $0.008-1$ & $0.008-0.5$ \\
\hline & GM & 0.43 & 4.2 & 0.32 & 75.66 & 0.08 & 0.08 & 0.15 \\
\hline & MIC90 & 1 & 16 & 1 & 256 & 0.125 & 0.5 & 0.25 \\
\hline & $\mathrm{R}$ & & $2.9 \%$ & & & 0 & $1.85 \%$ & 0 \\
\hline \multirow{3}{*}{$\begin{array}{l}\text { Saccharomyces cerevisiae } \\
n=12\end{array}$} & Range & $0.03-4$ & $0.06-8$ & $0.125-32$ & $0.25-256$ & $0.015-0.5$ & $0.08-0.5$ & $0.015-0.5$ \\
\hline & GM & 0.18 & 0.21 & 0.78 & 53.20 & 0.14 & 0.10 & 0.18 \\
\hline & MIC90 & 1 & 1 & 8 & 256 & 0.5 & 0.25 & 0.25 \\
\hline \multirow{4}{*}{$\begin{array}{l}\text { C. guilliermondii } \\
n=2\end{array}$} & Range & $0.06-0.5$ & $0.125-0.25$ & $0.25-0.5$ & $64-128$ & $0.015-0.25$ & $0.25-0.5$ & $0.015-0.25$ \\
\hline & MIC50 & 0.06 & 0.125 & 0.25 & 64 & 0.015 & 0.25 & 0.015 \\
\hline & MIC90 & 0.5 & 0.25 & 0.5 & 128 & 0.25 & 0.5 & 0.25 \\
\hline & R & & 0 & & & 0 & 0 & 0 \\
\hline $\begin{array}{l}\text { C. Iusitaniae } \\
n=1\end{array}$ & Range & 0.125 & 0.125 & 0.25 & 0.25 & 0.03 & 0.25 & 0.015 \\
\hline \multirow{3}{*}{$\begin{array}{l}\text { C. fabianii } \\
n=3\end{array}$} & Range & $0.06-0.25$ & 0.125 & 0.25 & 128 & $0.015-0.03$ & $0.015-0.25$ & $0.015-0.06$ \\
\hline & MIC50 & 0.06 & 0.125 & 0.25 & 128 & 0.015 & 0.015 & 0.03 \\
\hline & MIC90 & 0.25 & 0.125 & 0.25 & 128 & 0.03 & 0.25 & 0.06 \\
\hline $\begin{array}{l}\text { Trichosporon asahii } \\
n=1\end{array}$ & Range & 0.125 & 1 & 0.25 & 128 & 0.015 & 0.5 & 0.015 \\
\hline \multirow{3}{*}{$\begin{array}{l}\text { Rhodotorula } \\
n=3\end{array}$} & Range & $0.03-0.5$ & 0.125 & $0.06-0.125$ & $8-256$ & $0.06-0.5$ & $0.25-0.5$ & $0.25-0.5$ \\
\hline & MIC50 & 0.03 & 0.125 & 0.06 & 8 & 0.06 & 0.25 & 0.25 \\
\hline & MIC90 & 0.5 & 0.125 & 0.125 & 256 & 0.5 & 0.5 & 0.5 \\
\hline \multirow{3}{*}{$\begin{array}{l}\text { Kodamaea ohmeri } \\
n=2\end{array}$} & Range & $0.125-1$ & 0.125 & 0.5 & 128 & 0.015 & $0.125-0.25$ & $0.015-0.03$ \\
\hline & MIC50 & 0.125 & 0.125 & 0.5 & 128 & 0.015 & 0.125 & 0.015 \\
\hline & MIC90 & 1 & 0.125 & 0.5 & 128 & 0.015 & 0.25 & 0.03 \\
\hline \multirow{3}{*}{$\begin{array}{l}\text { Issatchenkia terricola } \\
n=2\end{array}$} & Range & $0.125-0.5$ & 8 & 0.5 & $128-256$ & 0.06 & 0.06 & 0.08 \\
\hline & MIC50 & 0.125 & 8 & 0.5 & 128 & 0.06 & 0.06 & 0.08 \\
\hline & MIC90 & 0.5 & 8 & 0.5 & 256 & 0.06 & 0.06 & 0.08 \\
\hline
\end{tabular}


Table 3 (continued)

\begin{tabular}{lllllllll}
\hline Candida species $(\mathbf{n})$ & \multicolumn{2}{l}{ Antifungal agents } & & & & \\
\cline { 2 - 9 } & & AmB & FLU & NYS & TEB & AFG & CFG & MFG \\
\hline $\begin{array}{l}\text { Torulasporapretoriensis } \\
n=1\end{array}$ & Range & 0.125 & 0.125 & 0.25 & 16 & 0.015 & 0.125 & 0.015 \\
ATCC90028 & Range & $0.03-2$ & $0.125-8$ & $0.25-16$ & $1-256$ & $0.008-0.015$ & $0.015-0.5$ & $0.008-0.015$ \\
& GM & 0.22 & 0.64 & 1.31 & 88.22 & 0.01 & 0.09 & 0.01 \\
& MIC90 & 1 & 2 & 8 & 256 & 0.015 & 0.5 & 0.015 \\
\hline
\end{tabular}

GM geometry mean, BUC butoconazole, CLO Clotrimazole, FLC Fluconazole, ITC Itraconazole, VRC Voriconazole, MIC Miconazole, TEC Terconazole, AmB Amphotericin B, FLU Flucytosine, NYS Nystatin, TEB Terbinafine, AFG Anidulafungin, CFG Caspofungin, MFG Micafungin

${ }^{a}$ ATCC90028 was tested 57 times

\section{Author details}

${ }_{1}^{1}$ Department of Obstetrics and Gynecology, Peking University Shenzhen Hospital, Shenzhen 518036, China. ${ }^{2}$ Shenzhen Key Laboratory of Gynecological Diagnostic Technology Research, Shenzhen 518036, China. ${ }^{3}$ Anhui Medical University, Hefei 230022, China. ${ }^{4}$ Department of Laboratory Science, Peking University Shenzhen Hospital, Shenzhen 518036, China.

Published online: 24 January 2022

\section{Reference}

1. Shi Yu, Zhu Y, Fan S, Liu X, Liang Y, Shan Y. Molecular identification and antifungal susceptibility profile of yeast from vulvovaginal candidiasis. BMC Infect Dis. 2020;20:287. https://doi.org/10.1186/ s12879-020-04985-w.

\section{Publisher's Note}

Springer Nature remains neutral with regard to jurisdictional claims in published maps and institutional affiliations. 